\title{
Groupoids, Hypergraphs, and Symmetries in Finite Models
}

\author{
Martin Otto \\ Department of Mathematics \\ Technische Universität Darmstadt
}

\begin{abstract}
We propose a novel construction of finite hypergraphs and relational structures that is based on reduced products with Cayley graphs of groupoids. The universal algebraic and combinatorial properties of groupoids are abstracted form the composition behaviour of partial injections and support a very natural approach to the construction of certain highly symmetric finite instances of hypergraphs and relational structures. The typical task of this kind asks for regular realisations of a locally specified overlap pattern between pieces (hyperedges, guarded substructures). We show that reduced products with groupoids provide a generic and versatile tool towards such constructions; they are explored in applications to the construction of finite hypergraph coverings, to finite model constructions for the guarded fragment, and to extension properties for partial isomorphisms of relational structures (in the sense of Hrushovski, Herwig, Lascar). To this end we construct groupoids whose Cayley graphs have large girth not just in the usual sense, but with respect to a discounted distance measure that contracts edges from the same sub-groupoid (colour) and only counts transitions between cosets (different colours), and show that their acyclicity properties guarantee corresponding degrees of acyclicity in reduced products.
\end{abstract}

\section{Key Notions And Results}

Consider a partial specification of some global structure by descriptions of its local constituents and of the possible links between these, in terms of allowed and required direct overlaps between pairs of local constituents. Such specifications typically have generic, highly regular, infinite realisations in the form of tree-like 'free' objects. We here address the issue of finite realisations, which should ideally meet similar criteria in terms of genericity, symmetry and specified degrees of acyclicity. We use hypergraphs as abstractions for the decomposition of global structures into local constituents and offer a versatile and generic solution to the finite synthesis problem posed by partial specifications of hypergraphs in terms of hyperedges and overlaps between these, w.r.t. natural criteria of controlled acyclicity in finite hypergraphs.

Hypergraphs are structures consisting of vertices that form vertex clusters called hyperedges; formally the hyperedges are just subsets of the vertex set. A typical hypergraph is denoted as $\mathfrak{A}=(A, S)$ with vertex set $A$ and set of hyperedges $S \subseteq$ $\mathcal{P}(A)$. Hypergraphs are the adequate combinatorial abstraction for various settings in which global structure is analysed in terms of local patches and their overlap pattern. Hypergraphs and overlap specifications between hyperedges arise, e.g., in connection with clusters of variables in CSP problems or conjunctive queries, as 'topological' abstractions of relational atoms in databases under certain constraints, as the orbits of sub-configurations under automorphisms of structures, and, generally, as patterns in all kind of structural decompositions. In the particular example of a 'topological' abstraction of the relations in a relational structure $\mathfrak{A}=(A,(R))$, consider the associated hypergraph $H(\mathfrak{A})=(A, S[\mathfrak{A}])$, where $S[\mathfrak{A}]$ consists of the guarded subsets of $\mathfrak{A}$, i.e., those subsets that are either singletons or a subset of a set $[\mathbf{a}]=\left\{a_{1}, \ldots, a_{k}\right\}$ of components of a tuple $\mathbf{a}=\left(a_{1}, \ldots, a_{k}\right)$ in one of the relations $R$ of $\mathfrak{A}$. This hypergraph of guarded subsets precisely captures the incidence pattern between the configurations in $\mathfrak{A}$ that are accessible to the guarded fragment GF of first-order logic, [2]; it has been extensively studied in connection with the model theory of GF as well as for many related algorithmic issues in database theory, see, e.g., [3] and references there.

Our main technical interest here is the analysis of local overlap specification versus global properties of finite hypergraphs - up to a natural notion of hypergraph bisimulation [13], [3] that arises, e.g., as the combinatorial abstraction of guarded bisimulation. This approach leads to the consideration of the local overlap specifications from a more generic point.

The structural information in a hypergraph $\mathfrak{A}=(A, S)$, with vertex set $A$ and set of hyperedges $S \subseteq \mathcal{P}(A)$ together with a labelling of the individual hyperedges, may be represented by a vertex- and edge-labelled graph structure $H=\left(V,\left(V_{s}\right)_{s \in S},\left(R_{e}\right)_{e \in E}\right)$. Intuitively, we pass to disjoint copies of the hyperedges and record their non-trivial overlaps by coloured matchings: the vertex set $V$ of $H$ is formed by the disjoint union $V:=\bigcup_{s \in S}\{s\} \times s$ of the hyperedges $s \in S$, partitioned by the unary predicates $V_{s}$ into the individual hyperedges $V_{s}=\{s\} \times s \subseteq V$; every pair of hyperedges $\left(s, s^{\prime}\right)$ with non-trivial intersection gives rise to an edge label $e=\left(s, s^{\prime}\right) \in E$ and a corresponding edge relation $R_{e} \subseteq V_{s} \times V_{s^{\prime}}$ prescribing the partial matching between $V_{s}$ and $V_{s^{\prime}}$ induced by the identity on $s \cap s^{\prime} \subseteq A$ : $R_{e}=\left\{\left((s, a),\left(s^{\prime}, a\right)\right): a \in s \cap s^{\prime}\right\}$. Viewing $I=(S, E)$ as the incidence pattern of the hypergraph, we slightly generalise this format to allow for more liberal specifications of desired overlap patterns, as follows.

Definition I.1. An incidence pattern is a finite directed loopfree multigraph $I=(S, E)$ with edges $e \in E\left[s, s^{\prime}\right]$ from $s$ to $s^{\prime}$, with an involutive, fixpoint-free converse operation $e \mapsto e^{-1}$ on $E$ that bijectively maps $E\left[s, s^{\prime}\right]$ to $E\left[s^{\prime}, s\right]$.

An $I$-graph with incidence pattern $I$ is a finite directed 
edge- and vertex-coloured graph $H=\left(V,\left(V_{s}\right)_{s \in S},\left(R_{e}\right)_{e \in E}\right)$ s.t. the vertex set $V$ is partitioned into subsets $V_{s}$ and, for $e \in E\left[s, s^{\prime}\right]$, the edge relation $R_{e} \subseteq V_{s} \times V_{s^{\prime}}$ induces a partial matching $\left(R_{e}\right.$ it is the graph of a partial 1-1 map $\rho_{e}$ from $V_{s}$ to $\left.V_{s^{\prime}}\right)$. The $\left(R_{e}\right)_{e \in E}$ are required to be compatible with the converse operation: $R_{e^{-1}}=\left(R_{e}\right)^{-1}$. An $I$-graph $H$ is complete if the $R_{e}$ induce full rather than partial matchings.

The representation of a hypergraph as discussed above is a special case of an incomplete $I$-graph, with the intersection graph $I(H)=(S, E)$, where $E=\left\{\left(s, s^{\prime}\right) \in S: s \neq s^{\prime}, s \cap\right.$ $\left.s^{\prime} \neq \emptyset\right\}$, as the underlying incidence pattern.

Among the most important criteria of structural simplicity in hypergraphs are acyclicity constraints - arising as natural albeit considerably more complex generalisations of graph acyclicity. The following criterion of hypergraph acyclicity is the natural notion for us (sometimes called $\alpha$-acyclicity), cf., e.g., [5], [4]. It is closely related to the algorithmically crucial notion of tree-decomposability (viz., existence of a tree-decomposition with hyperedges as bags) and to natural combinatorial notions of triangulation. It refers to the Gaifman graph $G(\mathfrak{A})$ associated with a hypergraph $\mathfrak{A}=(A, S)$, which has the same vertex set $A$ and links two distinct vertices by an undirected edge if they are elements of the same hyperedge.

Definition I.2. A finite hypergraph $\mathfrak{A}=(A, S)$ is acyclic if it is conformal and chordal:

(i) conformality: every clique in the Gaifman graph $G(\mathfrak{A})$ is contained in some hyperedge $s \in S$;

(ii) chordality: every cycle in the Gaifman graph $G(\mathfrak{A})$ of length greater than 3 has a chord.

For $N \geqslant 3, \mathfrak{A}=(A, S)$ is $N$-acyclic if it is $N$-conformal and $N$-chordal, where $N$-conformality $/ N$-chordality are the natural restrictions of the above to cliques/cycles of size $\leqslant N$.

Note that a hypergraph is $N$-acyclic if, and only if, every induced sub-hypergraph of up to $N$ vertices is acyclic.

Hypergraph coverings reproduce the overlap pattern between hyperedges of a given hypergraph in a covering hypergraph while smoothing out the overall behaviour, e.g., by achieving a higher degree of acyclicity.

In graphs one would without ambiguity appeal to local pattern versus global structure: local structure manifests itself in the incidence degrees of edges in individual vertices, while global structure manifests itself, e.g., in the length of the shortest cycles, also called the girth of the graph. In hypergraphs the situation is more complicated because the boundary between local and global aspects is blurred by the fact that the transition from one hyperedge to the next typically preserves several vertices while exchanging others.

Example I.3. Consider the full 3-uniform hypergraph on a set of 4 vertices - also familiar as the boundary of the 3simplex, or the faces of the tetrahedron, cf. Figures 1 and 2. We see that every vertex is incident with a 3-cycle of those three faces that share this vertex. The transitions between these faces each involve the exchange of just one vertex

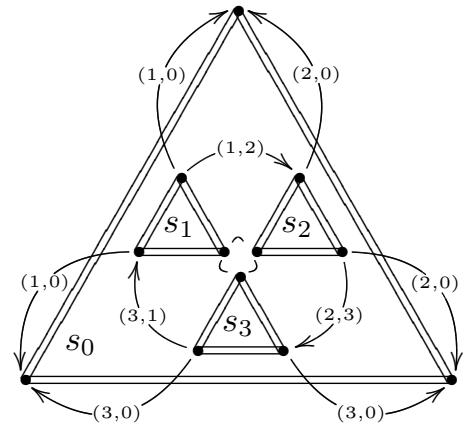

Fig. 1. Overlap specification for the tetrahedron.
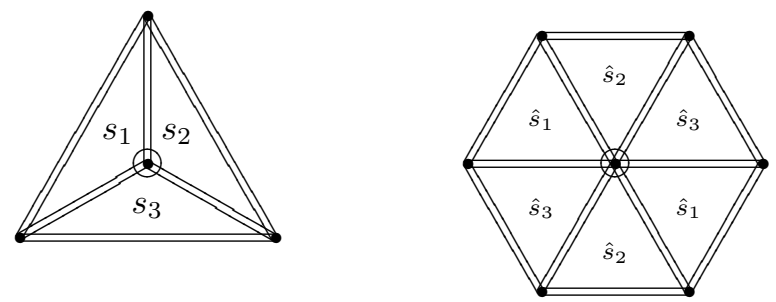

Fig. 2. Local 2-fold unfolding of the tetrahedron.

while fixing a pair of vertices. Any natural process of finite unfolding will locally produce a $3 n$-cycle instead of the 3 cycle, still centred on a shared vertex, and thus finite coverings cannot even locally avoid cycles (cf. Figure 2). Moreover, even this local view informs us that non-trivial coverings will affect incidence degrees (topologically we need to deal with branched coverings).

Both obstacles we see in this simple example, viz., the lack of a clear local-global distinction and the impossibility to preserve incidence degrees, generally seem to stand in the way of a straightforward construction of finite $N$-acyclic hypergraph covers - but neither arises in the special case of graphs.

As shown in [12], every finite graph admits, for each $N \in$ $\mathbb{N}$, a degree-preserving (topologically unbranched) covering by a finite graph without cycles of length up to $N$ (i.e., of girth greater than $N$ ). The generic construction of such $N$-acyclic graph coverings in [12] is based on a natural product of the given graph with Cayley groups of large girth. The latter can be obtained as subgroups of the symmetric groups of the vertex sets of suitably coloured finite acyclic graphs in an elegant construction due to Biggs [6], cf. Alon's survey [1].

Some of these ideas were successfully lifted and applied to the construction of hypergraph coverings in [13]. One part of the generalisation concerns a uniform construction of Cayley groups that not only have large girth in the usual sense; instead, they have large girth even w.r.t. to a reduced distance measure that measures the length of cycles in terms of the number of non-trivial transitions between cosets w.r.t. subgroups generated by different collections of generators. For an intuitive idea how this concern arises we may again look at the above example of the faces of the tetrahedron. 
There are two distinct sources of avoidable short cycles in its coverings: (a) 'local cycles' around a single pivot vertex; (b) 'non-local cycles' that enter and leave the 1-neighbourhoods of several distinct vertices. To account for the length of a cycle of type (b), the number of individual single-step transitions between faces around one of the visited pivotal vertices is typically irrelevant; what essentially matters is how often we move from one pivot to the next, and this corresponds to a transition between two subgroups (think of a transition between the stabiliser of one pivot and the next).

But nothing as simple as a (reduced) product between a hypergraph and even one of these 'highly acyclic' Cayley graphs will produce a covering by a finite $N$-acyclic hypergraph. The construction presented in [13] uses such Cayley groups only as one ingredient to achieve suitable coverings through an intricate local-to-global construction that is otherwise no longer canonical or natural, in the sense that it does not preserve symmetries of the given hypergraph.

We now expand the amalgamation techniques that were explored for the combinatorial construction in [13] of highly acyclic Cayley graphs from groups to groupoids and obtain 'Cayley groupoids' that are highly acyclic in a similar sense. It turns out that groupoids are a much better fit for the task of constructing hypergraph coverings as well as for the construction of finite hypergraphs according to other specifications. The new notion of Cayley groupoids allows for the construction of coverings by means of natural reduced products with these groupoids. It is more canonical and supports coverings of far greater genericity and symmetry than previously available. It also allows for substantial adaptations and generalisations. We address the covering problem and some variations and prove the following main theorems.

The first main theorem involves the natural notion of hypergraph coverings, as intuitively discussed above.

Definition I.4. A map $h: \hat{\mathfrak{A}} \rightarrow \mathfrak{A}$ between hypergraphs $\hat{\mathfrak{A}}=$ $(\hat{A}, \hat{S})$ and $\mathfrak{A}=(A, S)$ is a hypergraph homomorphism if, for every $\hat{s} \in \hat{S}, h\lceil\hat{s}$ is a bijection between the hyperedge $\hat{s}$ and some target hyperedge $h(\hat{s})$ of $\mathfrak{A}$.

A hypergraph covering of $\mathfrak{A}=(A, S)$ by $\hat{\mathfrak{A}}=(\hat{A}, \hat{S})$ is a hypergraph homomorphism $h: \hat{\mathfrak{A}} \rightarrow \mathfrak{A}$ that satisfies the backproperty w.r.t. hyperedges: for every $h(\hat{s})=s \in S$ and $s^{\prime} \in S$ there is some $\hat{s}^{\prime} \in \hat{S}$ such that $h\left(\hat{s}^{\prime}\right)=s^{\prime}$ and $h\left(\hat{s} \cap \hat{s}^{\prime}\right)=s \cap s^{\prime}$.

Theorem I.5. Every finite hypergraph admits, for every $N \in$ $\mathbb{N}$, a covering by a finite hypergraph that is $N$-acyclic. In addition, the covering hypergraph can be chosen to preserve all symmetries of the given hypergraph.

For details we refer to Section III and especially to Proposition III.2. The second main theorem addresses the more general issue of realising an abstract overlap specification in a finite hypergraph. A 'realisation' is required to provide locally all the overlaps as specified by the matchings in an $I$-graph $H$, yet globally to produce no other overlaps than those that are necessarily induced by compositions of those matchings.

To this end we extract from an incidence pattern $I=(S, E)$ the sets $E^{*}[s, t]$ of all words that arise as label sequences along directed paths from $s$ to $t$ in $I$. In an $I$-graph $H=$ $\left(V,\left(V_{s}\right),\left(R_{e}\right)\right)$ with partial matchings $\rho_{e}$ specified by the $R_{e}$ for $e \in E$, any $w \in E^{*}[s, t]$ induces a composition $\rho_{w}$ that is a partial bijection between $V_{s}$ and $V_{t}$ (possibly empty). In this context we may define a realisation of the overlap pattern specified by $H$ as follows.

Definition I.6. A hypergraph $\hat{\mathfrak{A}}=(\hat{A}, \hat{S})$ is a realisation of the overlap pattern specified by $H$, if there is a map $\pi: \hat{S} \rightarrow S$ and a matching family of bijections

$$
\pi_{\hat{s}}: \hat{s} \longrightarrow V_{s}, \text { for } \hat{s} \in \hat{S} \text { with } \pi(\hat{s})=s,
$$

such that for all $e \in E\left[s, s^{\prime}\right], \hat{s}, \hat{t} \in \hat{S}$ with $\pi(\hat{s})=s$ and $\pi(\hat{t})=t$ :

(i) there is some $\hat{s}^{\prime}$ such that $\pi\left(\hat{s}^{\prime}\right)=s^{\prime}$ and $\rho_{e}=\pi_{\hat{s}^{\prime}} \circ \pi_{\hat{s}}^{-1}$;

(ii) if $\hat{s} \cap \hat{t} \neq \emptyset$, then $\pi_{\hat{t}} \circ \pi_{\hat{s}}^{-1}=\rho_{w}$ for some $w \in E_{s t}^{*}$.

Theorem I.7. Every abstract finite specification of an overlap pattern between disjoint sets by an I-graph $H$ admits a finite realisation by a finite hypergraph, which may again be required to be $N$-acyclic and to preserve all symmetries of the given specification.

For details we refer to Section III-C and Proposition III.6. As corollaries we obtain new proofs of the theorems of Herwig [8] and Herwig and Lascar [9], which themselves have interesting applications to the finite model theory of the guarded fragment GF, [2], [7]. One of these applications also yields a new approach to a strong finite model property from [3], for GF in the presence of homomorphism constraints. While our new constructions do not achieve the good size bounds of [3] (essential there for the complexity of query answering), we achieve more symmetric models in a more generic and uniform treatment.

Structure of the paper: This presentation of the new techniques is meant to highlight the key notions and the more generic constructions. A more fully expanded account of the core material is given in [14], and much of the analysis of further applications has to be relegated to further study. Some of the core definitions, which have already been stated in this section for the statement of main results, will be taken up in the following sections. Section II introduces the notion of Cayley groupoids and presents a combinatorial method for their construction that is inspired by the work on Cayley groups in [13]. Section III presents hypergraph constructions based on natural reduced products with Cayley groupoids and shows how the acyclicity criteria achieved for groupoids in Section II translate into degrees of acyclicity, e.g., in finite hypergraph coverings. Section IV, finally, discusses the symmetries of hypergraphs constructed as reduced products with Cayley groupoids; there we also look at Herwig-Lascar extensions of partial isomorphism, and applications to the guarded fragment. 


\section{I-GROUPOIDS AND THEIR CAYLEY GRAPHS}

\section{A. I-groupoids}

Let $I=(S, E)$ be an incidence pattern (cf. Definition I.1), w.l.o.g. connected. An $I$-groupoid is generated by the labelled edges $e \in E$ of $I$, i.e., by the union of the sets $E\left[s, s^{\prime}\right]$ as specified in $I$. We are interested in the groupoidal structure of the composition of partial matchings in $I$-graphs. The main difference between groups and groupoids for our purposes is that the groupoidal operation is not total but only allows certain compositions, based on the sorts of elements. Correspondingly, groupoid elements carry labels that determine sorts and composability. We use $I$ and the sets $E^{*}[s, t]$ of labellings of paths in $I$ as the template for the groupoidal structure. Let $E^{*}$ stand for the union of the disjoint sets $E_{s t}^{*}$ consisting of the labellings of paths from $s$ to $t$ in $I$. For each $s \in S$, denote the corresponding empty word by $\lambda_{s} \in E_{s s}^{*}$. If $w=e_{1} \ldots e_{n} \in E_{s t}^{*}$ we write $w^{-1}:=e_{n}^{-1} \ldots e_{1}^{-1}$ for the converse in $E_{t s}^{*}$, which is obtained by reverse reading $w$ and simultaneously replacing each $e$ by its converse $e^{-1}$. The set $E^{*}$ carries a partially defined associative concatenation operation

$$
\left(w, w^{\prime}\right) \in E_{s t}^{*} \times E_{t u}^{*} \quad \longmapsto \quad w w^{\prime} \in E_{s u}^{*},
$$

which has the empty words $\lambda_{s} \in E_{s s}^{*}$ as neutral elements. One may think of this structure as a groupoidal analogue of the familiar word monoids. We refer to $\mathfrak{I}^{*}=$ $\left(E^{*},\left(E_{s t}^{*}\right)_{s, t \in S}, \cdot,\left(\lambda_{s}\right)_{s \in S}\right)$ as the free I-structure.

Definition II.1. An $S$-groupoid is a structure $\mathbb{G}=$ $\left(G,\left(G_{s t}\right)_{s, t \in S}, \cdot,\left(1_{s}\right)_{s \in S}\right)$ whose domain $G$ is partitioned into the sets $G_{s t}$, with designated $1_{s} \in G_{s s}$ and a partial binary operation ', which is precisely defined on the union of the sets $G_{s t} \times G_{t u}$ (with values in $G_{s u}$ ), such that the following are satisfied:

(i) (associativity) for all $g \in G_{s t}, h \in G_{t u}, k \in G_{u v}$ : $g \cdot(h \cdot k)=(g \cdot h) \cdot k$.

(ii) (neutral elements) for all $g \in G_{s t}: g \cdot 1_{t}=g=1_{s} \cdot g$.

(iii) (inverses) for every $g \in G_{s t}$ there is some $g^{-1} \in G_{t s}$ such that $g \cdot g^{-1}=1_{s}$ and $g^{-1} \cdot g=1_{t}$.

$\mathbb{G}$ is an $I$-groupoid if it is generated by a family $\left(g_{e}\right)_{e \in E}$ of groupoid elements associated with the edge labels $e \in E$, in the sense that $g_{e} \in G_{s s^{\prime}}$ for $e \in E\left[s, s^{\prime}\right], g_{e^{-1}}=\left(g_{e}\right)^{-1}$, and that every $g \in G_{s t}$ is represented by a product $\prod_{i=1}^{n} g_{e_{i}}$, for some $w=e_{1} \ldots e_{n} \in E_{s t}^{*}{ }^{1}$

In other words, an $I$-groupoid is a groupoid that is a homomorphic image of the free $I$-structure $\mathfrak{I}^{*}$, under the map

$$
\begin{aligned}
\mathbb{G}: \mathfrak{I}^{*} & \longrightarrow \mathbb{G} \\
w=e_{1} \ldots e_{n} \in E_{s t}^{*} & \longmapsto w^{\mathbb{G}}:=\prod_{i=1}^{n} g_{e_{i}} \in G_{s t} .
\end{aligned}
$$

For disconnected $I$, an $I$-groupoid breaks up into connected components that form separate groupoids, viz., one $I^{\prime}$-groupoid for each connected component $I^{\prime}$. For a subset

\footnotetext{
${ }^{1}$ It will make sense to identify the generator $g_{e}$ with $e$ itself, and we shal often also speak of groupoids generated by the family $(e)_{e \in E}$.
}

$\alpha=\alpha^{-1} \subseteq E$ that is closed under converse we denote by $\mathbb{G}_{\alpha}$ the sub-groupoid generated by $\left(g_{e}\right)_{e \in \alpha}$ within $\mathbb{G}$ :

$$
\mathbb{G}_{\alpha}=\mathbb{G} \uparrow\left\{w^{\mathbb{G}}: w \in \bigcup_{s t} \alpha_{s t}^{*}\right\} \text { with generators }\left(g_{e}\right)_{e \in \alpha},
$$

which may break up into separate and disjoint $I_{\alpha^{\prime}}$-groupoids for the disjoint connected components $I_{\alpha^{\prime}}$ of $I_{\alpha}$.

Consider an $I$-graph $H=\left(V,\left(V_{s}\right),\left(R_{e}\right)\right)$ (cf. Definition I.1). The partial bijections prescribed by the relations $R_{e}$, together with their compositions along paths in $E^{*}$, induce a structure of the same type as the free $\mathfrak{I}^{*}$, in fact a natural homomorphic image of $\mathfrak{I}^{*}$. For $e \in E\left[s, s^{\prime}\right]$, let $\rho_{e}$ be the partial bijection between $V_{s}$ and $V_{s^{\prime}}$ induced by $R_{e} \subseteq V_{s} \times V_{s^{\prime}}$. For $w \in E_{s t}^{*}$, define $\rho_{w}$ as the partial bijection from $V_{s}$ to $V_{t}$ induced by the composition of the maps $\rho_{e_{i}}$ along the path $w=e_{1} \ldots e_{n}$. For $w \in E_{s t}^{*}, \rho_{w}: V_{s} \rightarrow V_{t}$ is a partial bijection, possibly empty. In this manner we obtain a homomorphic image of the free $I$-structure $\mathfrak{I}^{*}=\left(E^{*},\left(E_{s t}^{*}\right) \cdot,\left(\lambda_{s}\right)\right)$. This homomorphism maps concatenation to (partial) composition:

$$
\rho_{w w^{\prime}}=\rho_{w^{\prime}} \circ \rho_{w} \quad \text { (composition of partial maps). }
$$

The converse operation $w \mapsto w^{-1}$ translates into inversion $\rho_{w} \mapsto \rho_{w^{-1}}=\left(\rho_{w}\right)^{-1}$ of partial maps, but in general this is not a groupoidal inverse. In fact it is the crucial distinguishing feature of complete $I$-graphs that we obtain a groupoidal inverse. More specifically, for an arbitrary $I$-graph $H$, the image of the free $I$-structure $\mathfrak{I}^{*}=\left(E^{*},\left(E_{s t}^{*}\right), \cdot,\left(\lambda_{s}\right)\right)$ under the homomorphism

$$
\begin{aligned}
\rho: \mathfrak{I}^{*} & \longrightarrow\{\rho: \rho \text { a partial bijection of } V\} \\
w=e_{1} \ldots e_{n} & \longmapsto \rho_{w}=\prod_{i=1}^{n} \rho_{e_{i}}
\end{aligned}
$$

produces a structure without groupoidal inverses. For a complete $I$-graph $H$, however, $\rho_{w^{-1}} \circ \rho_{w}=\operatorname{id}_{V_{s}}$ for any $w \in E_{s t}^{*}$, and the image structure obtained in this manner is a groupoid, which we denote as $\operatorname{cym}(H)$ :

$$
\rho: \mathfrak{I}^{*} \longrightarrow \operatorname{cym}(H)=\left(G,\left(G_{s t}\right)_{s, t \in S}, \cdot,\left(1_{s}\right)_{s \in S}\right)
$$

with $G_{s t}=\left\{\rho_{w}: w \in E_{s t}^{*}\right\}$, groupoid operation - as imposed by the natural composition structure between elements of matching sorts, and for $s \in S$, the identity $1_{s}=\operatorname{id}_{V_{s}}$ as the neutral element of sort $G_{s s}$. It is clear from the discussion above that $\rho_{w} \in G_{s t} \mapsto\left(\rho_{w}\right)^{-1}:=\rho_{w^{-1}} \in G_{t s}$ serves as the natural groupoidal inverse.

Definition II.2. For a complete $I$-graph $H$ we let $\operatorname{cym}(H)$ be the groupoid abstracted from $H$ according to the above stipulations. We consider $\operatorname{cym}(H)$ as an $I$-groupoid generated by $\left(\rho_{e}\right)_{e \in E}$.

We turn to the groupoidal analogue of the notion of the Cayley graph of a group.

Definition II.3. Let $\mathbb{G}=\left(G,\left(G_{s t}\right), \cdot,\left(1_{s}\right)\right)$ be an $I$-groupoid generated by $\left(g_{e}\right)_{e \in E}$. The Cayley graph of $\mathbb{G}$ is the complete $I$-graph $\mathbb{G}=\left(V,\left(V_{s}\right),\left(R_{e}\right)\right)$ where $V=G$,

$$
\begin{aligned}
V_{s} & =G_{* s}:=\bigcup_{t} G_{t s}, \\
R_{e} & =\left\{(g, g \cdot e): g \in V_{s}\right\} \text { for } e \in E\left[s, s^{\prime}\right] .
\end{aligned}
$$


One checks that the Cayley graph of an $I$-groupoid is a complete $I$-graph, in fact a very homogeneous one: the isomorphism type of the pointed $I$-graph $(\mathbb{G}, g)$ only depends on the 'sort' $s$, for which $g \in V_{s}=G_{* s}=\bigcup_{t} G_{t s}$. It also follows that the Cayley graph consists of a disjoint union of isomorphic complete $I$-graphs induced on the subsets $G_{t *}=\bigcup_{s} G_{t s}$ for $t \in S$ (if $I$ is connected, then these are also the connected components); the groupoid structure of $\mathbb{G}$ can be retrieved from each one of these, via cym.

Lemma II.4. The I-groupoid induced by the Cayley graph of $\mathbb{G}$ is isomorphic to $\mathbb{G}$.

We shall often just identify a groupoid with its Cayley graph. We thus find that the generic process of obtaining $I$-groupoids from complete $I$-graphs trivially reproduces the given $I$-groupoid when applied to such. We extend this process to the setting of not necessarily complete $I$-graphs by combining it with a process of completion.

\section{B. Completion of I-graphs}

If $H=\left(V,\left(V_{s}\right),\left(R_{e}\right)\right)$ is an $I$-graph, then the following produces a complete $I$-graph $H \times I$ on the vertex set $V \times S$ with the partition induced by the natural projection:

$$
\begin{aligned}
& V_{s}= V \times\{s\}, \text { and, for } e \in E\left[s, s^{\prime}\right], \\
& R_{e}=\left\{\left((v, s),\left(v^{\prime}, s^{\prime}\right)\right):\left(v, v^{\prime}\right) \text { an } e \text {-edge }\right\} \cup \\
&\left\{\left(\left(v^{\prime}, s\right),\left(v, s^{\prime}\right)\right):\left(v, v^{\prime}\right) \text { an } e \text {-edge }\right\} \cup \\
&\left\{\left((v, s),\left(v, s^{\prime}\right)\right): v \text { not incident with an } e \text {-edge }\right\} .
\end{aligned}
$$

The natural embedding $\sigma: v \mapsto(v, s)$ for $v \in V_{s}$ embeds $H$ isomorphically onto an induced substructure of $H \times I$. We often identify $H$ with $\sigma(H) \subseteq H \times I$. We use, as a completion of $H$, the relevant connected components of $H \times I$.

Definition II.5. The completion $\bar{H}$ of a not necessarily complete $I$-graph $H=\left(V,\left(V_{s}\right),\left(R_{e}\right)\right)$ is the union of the connected components in $H \times I$ that are incident with $\sigma(H)$.

Observation II.6. For every I-graph $H$, the completion $\bar{H}$ is a complete I-graph. Completion is compatible with disjoint unions: if $H=H_{1} \cup H_{2}$ is a disjoint union of I-graphs $H_{i}$, then $\bar{H}=\bar{H}_{1} \dot{\cup} \bar{H}_{2}$. If $H$ itself is complete, then $\bar{H} \simeq H$.

With the completion $H \mapsto \bar{H}$ we close the gap between $I$ graphs and induced $I$-groupoids. Cf. Definitions II.2 and II.5.

Definition II.7. For a not necessarily complete $I$-graph $H$, we let the induced $I$-groupoid $\operatorname{cym}(H)$ be the $I$-groupoid $\operatorname{cym}(\bar{H})$ induced by the completion of $H$.

The point is that the passage from $H$ to $\operatorname{cym}(H)$ produces an $I$-groupoid for any $I$-graph $H$, and reproduces the given $I$-groupoid when applied to its Cayley graph.

Connected components w.r.t. subsets of the edge colours $E$ will become important below. If $\alpha=\alpha^{-1} \subseteq E$ we write $I_{\alpha}$ for the reduct of $I$ to its $\alpha$-edges. We regard the $\alpha$ reducts of $I$-graphs (literally: their reducts to just those binary relations $R_{e}$ for $e \in \alpha$ ) as $I_{\alpha}$-graphs; the $\alpha$-reduct of the $I$ graph $H$ is denoted $H\left\lceil\alpha\right.$. An $I_{\alpha}$-graph may alternatively be regarded as an $I$-graph, with $R_{e}=\emptyset$ for all $e \notin \alpha$. Connected components of $I$-graphs w.r.t. $\alpha$-edges will arise as typical $I_{\alpha}$-graphs in some constructions. The following is immediate from the definitions.

Lemma II.8. Let $\alpha=\alpha^{-1} \subseteq E$, and consider an I-graph $H$ and its $\alpha$-reduct $K=H\lceil\alpha$, as well as their closures as $I$-graphs, $\bar{H}$ and $\bar{K}$, and the closure of $K$ as an $I_{\alpha}$-graph, $\bar{K}^{\alpha}$. Then $\bar{K}^{\alpha} \simeq \bar{K}\lceil\alpha \simeq \bar{H}\lceil\alpha$.

\section{Amalgamation of I-graphs}

The key to highly acyclic groupoids is the passage from $I$-graphs $H$ that contain unfoldings of short cycles formed by overlapping Cayley graphs of small sub-groupoids of $\mathbb{G}$ to the groupoid $\mathbb{G}^{*}:=\operatorname{cym}(H)$, which can no longer have those cycles. This technique is a non-trivial groupoidal analogue of the method expounded for Cayley groups in [13].

We want to consider local overlaps between Cayley graphs of sub-groupoids as follows. Let $\mathbb{G}_{\alpha}$ and $\mathbb{G}_{\beta}$ be two subgroupoids of an $I$-groupoid $\mathbb{G}$ with generators $e \in E$, where $\alpha=\alpha^{-1}, \beta=\beta^{-1} \subseteq E$ are closed under converse. We write $\mathbb{G}_{\alpha \beta}$ for $\mathbb{G}_{\alpha \cap \beta}$ and note that $\alpha \cap \beta$ is automatically closed under converse. For $g \in G_{* s}$ we may think of the connected component of $g$ in the reduct of the Cayley graph of $\mathbb{G}$ to $\left(R_{e}\right)_{e \in \alpha}$ as the $\mathbb{G}_{\alpha}$-coset at $g$ :

$$
g \mathbb{G}_{\alpha}=\left\{g \cdot w^{\mathbb{G}}: w \in \bigcup_{t} \alpha_{s t}^{*}\right\} \subseteq G .
$$

If $I_{\alpha}$ is connected, then $g \mathbb{G}_{\alpha}$, as a weak subgraph of the Cayley graph of $\mathbb{G}$, carries the structure of a complete $I_{\alpha}$ graph. If $I_{\alpha}$ consists of disjoint connected components, then $g \mathbb{G}_{\alpha}$ really produces the coset w.r.t. $\mathbb{G}_{\alpha^{\prime}}$ where $\alpha^{\prime}$ is the edge set of the connected component of $s$ in $I_{\alpha}$. In any case, this $I_{\alpha}$-graph is isomorphic to the connected component of $1_{s}$ in the Cayley graph of $\mathbb{G}_{\alpha}$.

Suppose the $I_{\alpha}$-graph $H_{\alpha}$ and the $I_{\beta}$-graph $H_{\beta}$ are disjoint but isomorphic to the Cayley graphs of sub-groupoids $\mathbb{G}_{\alpha}$ and $\mathbb{G}_{\beta}$, respectively. If $v_{1} \in H_{\alpha}$ and $v_{2} \in H_{\beta}$ are vertices of the same sort $s \in S$, then the connected components w.r.t. edge colours in $\alpha \cap \beta$ of $v_{1}$ in $H_{\alpha}$ and of $v_{2}$ in $H_{\beta}$ are related by a unique isomorphism. We define the amalgamation of $\left(H_{\alpha}, v_{1}\right)$ and $\left(H_{\beta}, v_{2}\right)$ to be the result of identifying the vertices in these two connected components in accordance with this unique isomorphism. It is convenient to speak of the subgroupoids $\mathbb{G}_{\alpha}$ as the constituents of such amalgams, but we keep in mind that we treat them as abstract $I$-graphs and not as embedded into $\mathbb{G}$. Let, in this sense, $\left(\mathbb{G}_{\alpha_{1}}, g_{1}\right) \oplus_{s}\left(\mathbb{G}_{\alpha_{2}}, g_{2}\right)$ stand for the amalgamation of the Cayley graphs of the two sub-groupoids $\mathbb{G}_{\alpha_{i}}$ in the vertices $g_{i} \in V_{s} \subseteq \mathbb{G}_{\alpha_{i}}$. Note that $\left(\mathbb{G}_{\alpha_{1}}, g_{1}\right) \oplus_{s}\left(\mathbb{G}_{\alpha_{2}}, g_{2}\right)$ is generally not a complete $I$-graph but satisfies the completeness requirement for edges $e \in \alpha_{1} \cap \alpha_{2}$.

Let $\left(\mathbb{G}_{\alpha_{i}}, g_{i}, h_{i}, s_{i}\right)_{1 \leqslant i \leqslant N}$ be a sequence of sub-groupoids with distinguished elements and vertex colours as indicated, and such that

$$
(\dagger)\left\{\begin{array}{l}
g_{i} \in\left(G_{\alpha_{i}}\right)_{* s_{i}} \subseteq \mathbb{G}_{\alpha_{i}} \\
h_{i} \in\left(G_{\alpha_{i}}\right)_{s_{i} s_{i+1}} \subseteq \mathbb{G}_{\alpha_{i}} \\
g_{i} \mathbb{G}_{\alpha_{i-1} \alpha_{i}} \cap g_{i} h_{i} \mathbb{G}_{\alpha_{i} \alpha_{i+1}}=\emptyset \text { as cosets in } \mathbb{G} .
\end{array}\right.
$$




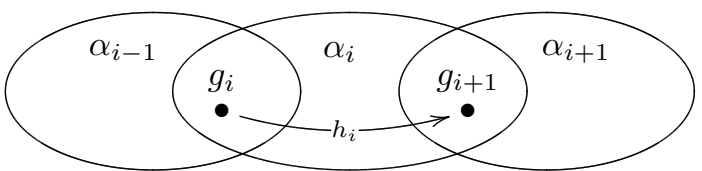

Fig. 3. Overlaps between cosets as in (unfolded) coset cycles.

Then the pairwise amalgams $\left(\mathbb{G}_{\alpha_{i}}, g_{i} h_{i}\right) \oplus_{s_{i}}\left(\mathbb{G}_{\alpha_{i+1}}, g_{i+1}\right)$ are individually well-defined and, due to the last requirement in $(\dagger)$, do not interfere. Together they produce a connected $I$-graph $H:=\bigoplus_{i=1}^{N}\left(\mathbb{G}_{\alpha_{i}}, g_{i}, h_{i}, s_{i}\right)$. We call this amalgam $a$ chain of sub-groupoids $\mathbb{G}_{\alpha_{i}}$ of length $N$.

\section{Avoiding short coset cycles}

Definition II.9. A coset cycle of length $n$ in an $I$-groupoid with generators $e \in E$ is a sequence $\left(g_{i}\right)_{i \in \mathbb{Z}_{n}}$ of groupoid elements $g_{i}$ (cyclically indexed) together with a sequence of colour sets $\alpha_{i}=\alpha_{i}^{-1} \subseteq E$ such that (as indicated in Fig. 3)

$h_{i}:=g_{i}^{-1} \cdot g_{i+1} \in \mathbb{G}_{\alpha_{i}}$ and $g_{i} \mathbb{G}_{\alpha_{i} \alpha_{i-1}} \cap g_{i+1} \mathbb{G}_{\alpha_{i} \alpha_{i+1}}=\emptyset$.

Definition II.10. An $I$-groupoid with generators $e \in E$ is $N$-acyclic if it does not have coset cycles of length up to $N$.

The following definition of compatibility captures the idea that some $I$-groupoid $\mathbb{G}$ is at least as discriminating as the $I$ groupoid $\operatorname{cym}(H)$ induced by an $I$-graph $H$. It also means that $\mathbb{G}=\operatorname{cym}(\mathbb{G})=\operatorname{cym}(\mathbb{G} \dot{\cup} H)-$ and in this role, compatibility of sub-groupoids $\mathbb{G}_{\alpha}$ with certain $H$ will serve as a guarantee for the preservation of these sub-groupoids in construction steps that render the overall $\mathbb{G}$ more discriminating.

Definition II.11. For an $I$-groupoid $\mathbb{G}$ and an $I$-graph $H$ we say that $\mathbb{G}$ is compatible with $H$ if, for every $s \in S$ and $w \in E_{s s}^{*}, w^{\mathbb{G}}=1_{s}$ implies $\rho_{w}=\mathrm{id}_{V_{s}}=1_{s}$ in $\operatorname{cym}(H)$, too.

The condition of compatibility is such that the natural homomorphisms for the free $\mathfrak{I}^{*}$ onto $\mathbb{G}$ and onto $\operatorname{cym}(H)$ induce a homomorphism from $\mathbb{G}$ onto $\operatorname{cym}(H)$, as in this commuting diagram:

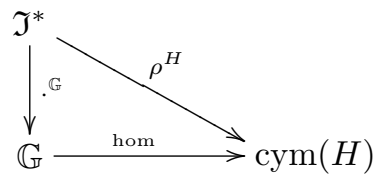

Remark II.12. For any I-graphs $K$ and $H, \operatorname{cym}(H \cup \dot{\cup} K)$ is compatible with $K, \bar{K}$ and with the Cayley graph of $\operatorname{cym}(K)$.

The following holds the key to avoiding short coset cycles. Note that in effect we only consider sub-groupoids generated by even generator sets, as we require closure under converse.

Lemma II.13. Let $\mathbb{G}$ be an I-groupoid with generators $e \in$ $E, k \in \mathbb{N}$, and assume that, for every $\alpha=\alpha^{-1} \subseteq E$ with $|\alpha|<2 k$, the sub-groupoid $\mathbb{G}_{\alpha}$ is compatible with chains of groupoids $\mathbb{G}_{\alpha \beta_{i}}$ up to length $N$, for any choice of subsets $\beta_{i}=\beta_{i}^{-1} \subseteq E$. Then there is a finite I-groupoid $\mathbb{G}^{*}$ s.t.

(i) for every $\alpha=\alpha^{-1} \subseteq E$ with $|\alpha|<2 k$, $\mathbb{G}_{\alpha}^{*} \simeq \mathbb{G}_{\alpha}$, and (ii) for all $\alpha=\alpha^{-1} \subseteq E$ with $|\alpha| \leqslant 2 k$, the sub-groupoid $\mathbb{G}_{\alpha}^{*}$ is compatible with chains $\mathbb{G}_{\alpha \beta_{i}}^{*}$ up to length $N$.

It will be important later that compatibility of $\mathbb{G}_{\alpha}^{*}$ with chains as in (ii) makes sure that $\mathbb{G}_{\alpha}^{*}$ cannot have coset cycles with colours $\alpha \cap \beta_{i}$ of length up to $N$, because every such cycle in the Cayley groupoid $\mathbb{G}_{\alpha}^{*}$ would have to be a cycle also in the Cayley groupoid induced by that chain, i.e., by the unfolding of the coset cycle under consideration, which is blatantly impossible.

Proof of the lemma: $\mathbb{G}^{*}$ is obtained as $\mathbb{G}^{*}:=\operatorname{cym}(H)$ for an $I$-graph $H=\mathbb{G} \dot{\cup} K$ consisting of the disjoint union of (the Cayley graph of) $\mathbb{G}$ and certain chains of sub-groupoids of $\mathbb{G}$. Specifically, we let $K$ be the disjoint union of all amalgamation chains of length up to $N$ of the form

$$
\bigoplus_{i=1}^{m}\left(\mathbb{G}_{\alpha \beta_{i}}, g_{i}, h_{i}, s_{i}\right)
$$

for $\alpha=\alpha^{-1}, \beta_{i}=\beta_{i}^{-1} \subseteq E, 1 \leqslant i \leqslant m \leqslant N$, where $|\alpha| \leqslant$ $2 k$. By construction and Remark II.12, $\mathbb{G}^{*}=\operatorname{cym}(\mathbb{G} \cup K)$ is compatible with chains $\mathbb{G}_{\alpha \beta_{i}}$ of the required format; together with (i) this implies (ii), i.e., that $\mathbb{G}^{*}$ is compatible with corresponding chains of $\mathbb{G}_{\alpha \beta_{i}}^{*}$. For (i), it suffices to show that, for $\left|\alpha^{\prime}\right|<2 k, \mathbb{G}_{\alpha^{\prime}}$ is compatible with each connected component of $K$. Compatibility with $\mathbb{G}$ is obvious. Consider then a component of the form $\bigoplus_{i=1}^{m}\left(\mathbb{G}_{\alpha \beta_{i}}, g_{i}, h_{i}, s_{i}\right)$. Its $\alpha^{\prime}$ components are obtained as merged chains of components of the form $\mathbb{G}_{\alpha^{\prime} \alpha \beta}$. Since $\left|\alpha^{\prime}\right|<2 k$, the assumptions of the lemma imply compatibility of $\mathbb{G}_{\alpha^{\prime}}$ with any such component. It follows that $\mathbb{G}^{*}=\operatorname{cym}(\mathbb{G} \cup K)$ is compatible with all $\mathbb{G}_{\alpha^{\prime}}$ for $\left|\alpha^{\prime}\right|<2 k$, and thus $\mathbb{G}_{\alpha^{\prime}}^{*} \simeq \mathbb{G}_{\alpha^{\prime}}$ for $\left|\alpha^{\prime}\right|<2 k$. Compare Remark II.12.

The proof of the following is then obtained by inductive application of Lemma II.13 for increasing $k$. We also state, without proof, an easy but crucial observation that clarifies the relationship between embedded sub-groupoids in $\mathbb{G}$.

Proposition II.14. For every incidence pattern $I=(S, E)$ and $N \in \mathbb{N}$ there are finite $N$-acyclic I-groupoids with generators $e \in E$.

Observation II.15. For any 2-acyclic I-groupoid $\mathbb{G}$ and any subsets $\alpha=\alpha^{-1}, \beta=\beta^{-1} \subseteq E$, with associated subgroupoids $\mathbb{G}_{\alpha}, \mathbb{G}_{\beta}$ and $\mathbb{G}_{\alpha \beta}: \mathbb{G}_{\alpha} \cap \mathbb{G}_{\beta}=\mathbb{G}_{\alpha \beta}$.

\section{HYPERGRAPH COVERINGS AND UNFOLDINGS}

Recall from Section I that we associate with a hypergraph $\mathfrak{A}=(A, S)$ its Gaifman graph $G(\mathfrak{A})=(A, G(S))$, which may be viewed as a superposition of cliques, one for each hyperedge; and its intersection graph $I(\mathfrak{A})=(S, E)$ as the induced incidence pattern. The induced sub-hypergraph $\mathfrak{A} \uparrow$ $A_{0}$ of a hypergraph $\mathfrak{A}=(A, S)$ is the hypergraph on $A_{0}$ with hyperedge set $S\left\lceil A_{0}:=\left\{s \cap A_{0}: s \in S\right\}\right.$. Recall the definition of hypergraph acyclicity and $N$-acyclicity in terms of conformality and chordality conditions from Definition I.2. If $\mathfrak{A}$ is $N$-acyclic, then every induced sub-hypergraph $\mathfrak{A} \uparrow A_{0}$ on subsets $A_{0} \subseteq A$ of size up to $N$ is acyclic. Also recall the 
definition of hypergraph coverings in Definition I.4 as special hypergraph homomorphisms with the back-property.

\section{A. Coverings by reduced products with groupoids}

Let $\mathfrak{A}=(A, S)$ be a finite hypergraph with intersection graph $I:=I(\mathfrak{A})=(S, E)$. Let $\mathbb{G}$ be an $I$-groupoid with generators $e \in E$. For $a \in A$ we let $\mathbb{G}_{a}$ denote the subgroupoid of $\mathbb{G}$ generated by $E_{a}:=\left\{\left(s, s^{\prime}\right) \in E: a \in s \cap s^{\prime}\right\}$.

We construct a natural covering of $\mathfrak{A}$,

$$
\pi: \mathfrak{A} \otimes \mathbb{G} \longrightarrow \mathfrak{A},
$$

where $\mathfrak{A} \otimes \mathbb{G}=(\hat{A}, \hat{S})$ is a reduced product of $\mathfrak{A}$ with $\mathbb{G}$ as follows. The vertex set $\hat{A}$ is the quotient of the disjoint union of hyperedges $s$ of $\mathfrak{A}$ tagged by groupoid elements $g \in G_{* s}$,

$$
\bigcup_{s \in S, g \in G_{* s}}\{g\} \times\{s\} \times s
$$

w.r.t. the equivalence relation induced by identifications

$$
(g, s, a) \approx\left(g e, s^{\prime}, a\right) \quad \text { for } e=\left(s, s^{\prime}\right) \in E_{a} .
$$

We note that $\left(g_{1}, s_{1}, a\right)$ is identified with $\left(g_{2}, s_{2}, a\right)$ in this quotient if, and only if, there is a path $w$ from $s_{1}$ to $s_{2}$ in $I$ consisting of edges $e=\left(s, s^{\prime}\right) \in E_{a}$, for which $a \in s \cap s^{\prime}$, and such that $g_{2}=g_{1} \cdot w^{\mathbb{G}}$. We think of the generators $e=$ $\left(s, s^{\prime}\right) \in E_{a}$ as preserving the vertex $a$ in passage from $a \in s$ to $a \in s^{\prime}$ : the $g$-tagged copy of $s$ and the $g^{\prime}$-tagged copy of $s^{\prime}$ are glued in their overlap $s \cap s^{\prime}$, for $g^{\prime}=g e$.

Let us denote the equivalence class of a triple $(g, s, a)$ as $[g, s, a]$. Then the hyperedges of $\mathfrak{A} \otimes \mathbb{G}=(\hat{A}, \hat{S})$ are the subsets represented by the natural copies of hyperedges $s \in S$ :

$$
\hat{S}=\left\{[g, s]: s \in S, g \in G_{* s}\right\}
$$

where $[g, s]=\{[g, s, a]: a \in s\} \subseteq \hat{A}$. The cover homomorphism $\pi$ is the natural projection $\pi:[g, s, a] \mapsto a$, which turns $\pi: \hat{\mathfrak{A}} \rightarrow \mathfrak{A}$ into a hypergraph covering according to Definition I.4. Crucially, $N$-acyclicity of $\mathbb{G}$ transfers to $N$ acyclicity of the coverings thus obtained. The proof of the key lemma is omitted here, but can be found in [14].

Lemma III.1. Let $\mathfrak{A}$ be a hypergraph with intersection graph $I(\mathfrak{A})=: I, \mathbb{G}$ an $N$-acyclic $I$-groupoid. Then $\mathfrak{A} \otimes \mathbb{G}$ is $N$ chordal and $N$-conformal.

Together with Proposition II.14 we obtain the following.

Proposition III.2. For every $N \in \mathbb{N}$, every finite hypergraph admits a covering by a finite hypergraph that is $N$-acyclic.

\section{B. Hypergraph unfoldings}

We explore a weaker analogue of coverings for situations where hyperedge overlaps are limited to specified subsets of the overlaps realised in the given hypergraph. This variation is of interest as an intermediary between coverings and realisations in the sense of Definition I.6. Technically we therefore look at modifications of the back-property in coverings from Definition I.4; and at specifications of overlaps in terms of a richer incidence pattern $I$ than the actual intersection graph. Intuitively, the set $d[e] \subseteq s \cap s^{\prime}$ for $e \in E\left[s, s^{\prime}\right]$ in the following definition is the domain in which copies of $s$ and $s^{\prime}$ are meant to overlap according to $e$.

Definition III.3. A link structure for a hypergraph $\mathfrak{A}=(A, S)$ is an incidence pattern $I=(S, E)$ together with a map $d$ associating with every edge $e \in E\left[s, s^{\prime}\right]$ and its converse $e^{-1} \in E\left[s^{\prime}, s\right]$ the same subset $d[e]=d\left[e^{-1}\right] \subseteq s \cap s^{\prime}$.

A hypergraph homomorphism $h: \hat{\mathfrak{A}} \rightarrow \mathfrak{A}$ is an $I$-covering if it satisfies the back-property w.r.t. links specified in $I$ : for every $h(\hat{s})=s \in S, s^{\prime} \in S$ and $e \in E\left[s, s^{\prime}\right]$ in $I$, there is some $\hat{s}^{\prime} \in \hat{S}$ such that $h\left(\hat{s}^{\prime}\right)=s^{\prime}$ and $h\left(\hat{s} \cap \hat{s}^{\prime}\right)=d[e] \subseteq s \cap s^{\prime}$.

We produce $I$-coverings as reduced products with $I$ groupoids. For a hypergraph $\mathfrak{A}$, link structure $I$ and an $I$ groupoid $\mathbb{G}$, we define a hypergraph

$$
\mathfrak{A} \otimes_{E} \mathbb{G}=(\hat{A}, \hat{S})
$$

as a reduced product in analogy with the above definition of $\mathfrak{A} \otimes \mathbb{G}$. Now $\left(g_{1}, s_{1}, a\right)$ is identified with $\left(g_{2}, s_{2}, a\right)$ if, and only if, $g_{2}=g_{1} \cdot w^{\mathbb{G}}$ for some path $w$ from $s_{1}$ to $s_{2}$ in $I$ consisting of edges $e$ for which $a \in d[e]$.

It is not hard to see, by arguments strictly analogous to those given for coverings in Section III-A, that $\mathfrak{A} \otimes_{E} \mathbb{G}$ is $N$-acyclic for $N$-acyclic $\mathbb{G}$.

Proposition III.4. Any finite hypergraph $\mathfrak{A}$ with specified link structure I admits, for any $N \in \mathbb{N}$, an I-covering by a finite $N$-acyclic hypergraph $\hat{\mathfrak{A}}$.

\section{Unfoldings of I-graphs and realisations}

We may also define a hypergraph $\tilde{\mathfrak{A}}:=H \otimes \mathbb{G}$ as a reduced product of an $I$-graph $H$ and $I$-groupoid $\mathbb{G}$. Its vertex set is the quotient of the disjoint union of the sets $V_{s}$ in $H$, tagged by groupoid elements $g \in G_{* s}$ and (redundantly) by $s$ itself, $\bigcup_{s \in S, g \in G_{* s}}\{g\} \times\{s\} \times V_{s}$ w.r.t. the equivalence relation $\approx$ obtained by identifying $(g, s, u)$ with $\left(g e, s^{\prime}, v\right)$ whenever $g \in$ $G_{* s}, e \in E\left[s, s^{\prime}\right]$ and $(u, v) \in R_{e}$. Note that, for $g_{i} \in G_{* s_{i}}$ and $v_{i} \in V_{s_{i}},\left(g_{1}, s_{1}, v_{1}\right)$ is identified with $\left(g_{2}, s_{2}, v_{2}\right)$ in the quotient if, and only if, there is a path $w \in E_{s_{1} s_{2}}^{*}$ such that $\rho_{w}\left(v_{1}\right)=v_{2}$ in $H$ and $g_{2}=g_{1} \cdot w^{\mathbb{G}}$ in $\mathbb{G}$. The hyperedges of $\tilde{\mathfrak{A}}$ are subsets represented by the natural copies of the patches $V_{s}$ of $H$. We then find the following.

Lemma III.5. If $\mathbb{G}$ is compatible with the I-graph $H=$ $\left(V,\left(V_{s}\right),\left(R_{e}\right)\right)$, then the natural projection $\pi_{[g, s]}:[g, v] \mapsto v$ is well-defined in restriction to each hyperedge $[g, s]$ of $H \otimes \mathbb{G}$, and relates the hyperedge $[g, s]=\left\{[g, s, v]: v \in V_{s}\right\}$ bijectively to $V_{s}$.

Consider an $I$-graph $H=\left(V,\left(V_{s}\right),\left(R_{e}\right)\right)$ as a specification of overlaps to be realised between isomorphic copies of the sets $V_{s}$ according to identifications induced by the partial matchings $R_{e}$ or $\rho_{e}$ of $H$. Compare Definition I.6 for the formal definition of a realisation. Reduced products $\tilde{\mathfrak{A}}:=H \otimes \mathbb{G}$ with $I$-groupoids $\mathbb{G}$ that are compatible with $H$ are a first approximation: according to Lemma III.5, the hyperedges of $H \otimes \mathbb{G}$ individually project bijectively onto the respective sets $V_{s}$. It is not clear, however, that hyperedges 
$\left[g_{1}, s\right]$ and $\left[g_{2}, t\right]$, related to $V_{s}$ and $V_{t}$, do not overlap to a greater extent than specified in $H$. To obtain a realisation, we apply an unfolding of the form $\hat{\mathfrak{A}}=\tilde{\mathfrak{A}} \otimes_{\tilde{E}} \tilde{\mathbb{G}}$ w.r.t. a suitable link structure $\tilde{I}=(\tilde{S}, \tilde{E})$ and a 2 -acyclic $\tilde{I}$-groupoid $\tilde{\mathbb{G}}$.

With $e \in E\left[s, s^{\prime}\right]$ (from the original multi-graph $I=(S, E)$ ) we associate edges $\tilde{e} \in \tilde{E}\left[\tilde{s}, \tilde{s}^{\prime}\right]$ from $\tilde{s}=[g, s] \in \tilde{S}$ to $\tilde{s}^{\prime}=\left[g \cdot e, s^{\prime}\right] \in \tilde{S}$. These hyperedges $\tilde{s}$ and $\tilde{s}^{\prime}$ have bijective projections $\pi_{\tilde{s}}:[g, s] \rightarrow V_{s}$ and $\pi_{\tilde{s}^{\prime}}:\left[g \cdot e, s^{\prime}\right] \rightarrow V_{s^{\prime}}$. We specify $\tilde{e} \in \tilde{E}\left[\tilde{s}, \tilde{s}^{\prime}\right]$ through $d[\tilde{e}]:=\left\{[g, s, v]: v \in \operatorname{dom}\left(\rho_{e}\right)\right\}$. A hyperedge of $\hat{\mathfrak{A}}$ is of the form $\hat{s}=[\tilde{g}, \tilde{s}]$ where $\tilde{g} \in \tilde{G}_{* \tilde{s}}$. From $\hat{\mathfrak{A}}=\tilde{\mathfrak{A}} \otimes_{\tilde{E}} \tilde{\mathbb{G}}$, this hyperedge $\hat{s}$ bijectively projects onto the hyperedge $\tilde{s}$ of $\tilde{\mathfrak{A}}=H \otimes \mathbb{G}$, and from there bijectively onto the appropriate $V_{s}$. As shown in [14], this method yields a realisation.

Proposition III.6. For every incidence pattern I and I-graph $H$, there is a finite hypergraph $\hat{\mathfrak{A}}$ that realises the overlap pattern specified by $H$. For a given threshold $N \in \mathbb{N}$, such realisations $\hat{\mathfrak{A}}$ can be chosen to be $\mathrm{N}$-acyclic.

\section{SYMMETRIES}

Hypergraph coverings $\hat{\mathfrak{A}}=\mathfrak{A} \otimes \mathbb{G}$, and similarly unfoldings and $I$-coverings, obtained as reduced products with $I$ groupoids $\mathbb{G}$, have characteristic 'vertical' symmetries that are compatible with the relevant projections. These symmetries are groupoidal in the sense that they are inherited from the structural homogeneity of the groupoid $\mathbb{G}$. Another kind of symmetries may arise from structural symmetries of the underlying hypergraph, link-structure, or $I$-graph. Both kinds of symmetries are described in terms of hypergraph automorphisms in the natural manner: bijections $\eta: A \rightarrow A$ that preserve $S$. We state without proof first the existence of vertical, groupoidal symmetries. Compare the remark after Definition II.3 regarding the symmetries of the Cayley graphs of groupoids.

Lemma IV.1. In a hypergraph covering $\pi: \mathfrak{A} \otimes \mathbb{G} \rightarrow \mathfrak{A}$ by a reduced product with any $I$-groupoid $\mathbb{G}$, where $I=I(\mathfrak{A})$, any two pre-images of the same hyperedge $s \in S$ are related by an automorphism of $\mathfrak{A} \otimes \mathbb{G}$ that commutes with $\pi$. Similarly, the reduced products $\mathfrak{A} \otimes_{E} \mathbb{G}$ and $H \otimes \mathbb{G}$ (for $\mathbb{G}$ compatible with $H)$, admit automorphisms that relate any given two hyperedges above the same hyperedge $s$ of $\mathfrak{A}$, or above the same $V_{s}$ of $H$, and are compatible with the projection $\pi: \mathfrak{A} \otimes_{E} \mathbb{G} \rightarrow \mathfrak{A}$, or with the local projections $\pi_{\hat{s}}: \hat{s} \rightarrow V_{s}$, respectively.

Other than these vertical symmetries within fibres, the compatibility of reduced products with automorphisms of the given structure relies on special symmetry properties of the groupoid $\mathbb{G}$, and ultimately of the incidence pattern $I$. Looking at symmetries of $I$ (not just plain automorphisms of the structure $I$ as we also want to permute edge labels), we can show that for suitably symmetric groupoids all our constructions are sufficiently canonical to lift all symmetries of a hypergraph $\mathfrak{A}$ to its coverings by $\mathfrak{A} \otimes \mathbb{G}$, of a hypergraph $\mathfrak{A}$ with specified link structure to its unfoldings $\mathfrak{A} \otimes_{E} \mathbb{G}$, of an $I$-graph $H$ to its unfolding $H \otimes \mathbb{G}$, and to its realisations. The precise technical definitions and arguments are presented in [14] and have to be omitted here. The definitions are just the natural ones. To give some indicative examples, a symmetry of an incidence pattern $I$ is an automorphism of the associated two-sorted incidence structure, i.e., a pair $\eta^{I}=\left(\eta^{S}, \eta^{E}\right)$ of bijections $\eta^{S}: S \rightarrow S$ and $\eta^{E}: E \rightarrow E$, such that $\eta^{E}(e) \in E\left[\eta^{S}(s), \eta^{S}(t)\right]$ iff $e \in E[s, t]$; and a symmetry of the link structure $I=(S, E)$ for a hypergraph $\mathfrak{A}=(A, S)$ is induced by a hypergraph automorphism $\eta^{\mathfrak{A}}: \mathfrak{A} \rightarrow \mathfrak{A}$ through the natural stipulations for $\eta^{S}: S \rightarrow S$ and $\eta^{E}: E \rightarrow E$ (mapping $e \in E\left[s, s^{\prime}\right]$ to $\eta(e) \in E\left[\eta(s), \eta\left(s^{\prime}\right)\right]$ where $d[\eta(e)]=\eta(d[e])$ through operation of $\eta$ on subsets $d \subseteq A$ ). Similarly, a symmetry of an $I$-groupoid $\mathbb{G}$ with generators $e \in E$ is a bijection $\eta^{\mathbb{G}}: G \rightarrow G$ with an induced symmetry $\eta^{I}=\left(\eta^{S}, \eta^{E}\right)$ of $I$, such that for all $e \in E\left[s, s^{\prime}\right], \eta^{\mathbb{G}}$ maps the generator $e \in G_{s s^{\prime}}$ to the generator $\eta(e) \in G_{\eta(s) \eta\left(s^{\prime}\right)}$ and, correspondingly, for all $s \in S$ and $g_{1} \in G_{s t}, g_{2} \in G_{t u}$ :

(i) $\eta^{\mathbb{G}}\left(1_{s}\right)=1_{\eta(s)}$

(ii) $\eta^{\mathbb{G}}\left(g_{1} \cdot g_{2}\right) \stackrel{=}{=} \eta^{\mathbb{G}}\left(g_{1}\right) \cdot \eta^{\mathbb{G}}\left(g_{2}\right)$.

An automorphism $\eta^{\mathfrak{A}}$ of a hypergraph $\mathfrak{A}$ induces a symmetry $\eta^{I}=\left(\eta^{S}, \eta^{E}\right)$ of $I=I(\mathfrak{A})$. If the $I$-groupoid $\mathbb{G}$ has a symmetry $\eta=\left(\eta^{\mathbb{G}}, \eta^{I}\right)$ with the same underlying symmetry $\eta^{I}$, then the cover $\pi: \mathfrak{A} \otimes \mathbb{G} \rightarrow \mathfrak{A}$ carries a corresponding symmetry that is both an automorphism of the covering hypergraph $\hat{\mathfrak{A}}=\mathfrak{A} \otimes \mathbb{G}$ and compatible with the given automorphism of $\mathfrak{A}$ in the sense of a commuting diagram

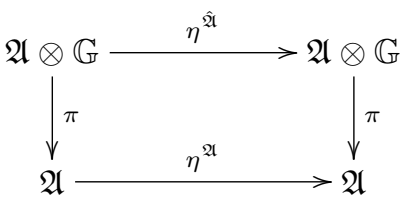

Upon re-inspection, the construction steps in the generation of $N$-acyclic $I$-groupoids towards Proposition II.14 are naturally compatible with any symmetry of the given $I$.

Corollary IV.2. If $I=(S, E)$ is an incidence pattern, then there is, for every $N \in \mathbb{N}$, some finite $N$-acyclic I-groupoid $\mathbb{G}$ whose symmetries induce all the symmetries of $I$.

From Proposition III. 2 we thus further obtain the following.

Corollary IV.3. Any finite hypergraph $\mathfrak{A}$ admits, for $N \in \mathbb{N}$, finite $N$-acyclic coverings by reduced products with finite $N$ acyclic I-groupoids, $\pi: \hat{\mathfrak{A}}=\mathfrak{A} \otimes \mathbb{G} \rightarrow \mathfrak{A}$, that are compatible with the automorphism group of $\mathfrak{A}$ in the sense that every automorphism $\eta^{\mathfrak{A}}$ of $\mathfrak{A}$ lifts to an automorphism $\eta^{\hat{\mathfrak{A}}}$ such that $\pi \circ \eta^{\hat{\mathfrak{A}}}=\eta^{\mathfrak{A}} \circ \pi$.

Similarly, and most importantly for further applications, reduced products $\mathfrak{A} \otimes_{E} \mathbb{G}$ with hypergraphs or $H \otimes \mathbb{G}$ with $I$-graphs support the same kinds of symmetries as $\mathfrak{A} \otimes \mathbb{G}$.

Corollary IV.4. Every symmetry $\eta$ of $(\mathfrak{A}, I, \mathbb{G})$ gives rise to an automorphism of $\mathfrak{A} \otimes_{E} \mathbb{G}$. Every symmetry $\eta$ of $(H, I, \mathbb{G})$ gives rise to an automorphism of $H \otimes \mathbb{G}$.

For any I-graph $H=\left(V,\left(V_{s}\right),\left(R_{e}\right)\right)$, realisations $\hat{\mathfrak{A}}$ as obtained in Proposition III.6 can be chosen so that all symmetries 


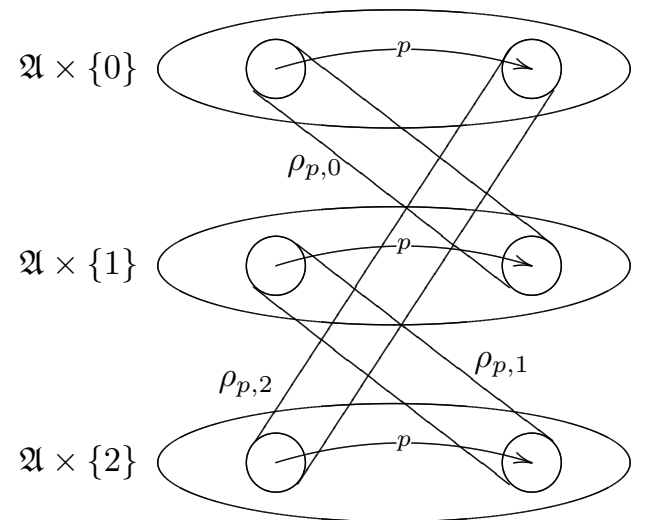

Fig. 4. Basic structure for Herwig extensions w.r.t. a single $p$.

of $H$ lift to automorphisms of $\hat{\mathfrak{A}}$. Moreover, for any two hyperedges $\hat{s}_{1}$ and $\hat{s}_{2}$ of $\hat{\mathfrak{A}}$ that bijectively project to the same $V_{s}$, there is a 'vertical' automorphism of $\hat{\mathfrak{A}}$ that is compatible with these projections.

\section{A. An application to Herwig's theorem and $G F$}

In its basic form, Herwig's theorem [8], [9] provides, for some given partial isomorphism $p$ of a finite relational structure $\mathfrak{A}$, an extension $\mathfrak{B} \supseteq \mathfrak{A}$ of $\mathfrak{A}$ such that the given partial isomorphism $p$ of $\mathfrak{A}$ extends to a full automorphism of $\mathfrak{B}$. It generalises a corresponding theorem about finite graphs by Hrushovski [11]. A substantial generalisation (cf. Corollary IV.9) is proved in the paper by Herwig and Lascar [9]. W.l.o.g. we restrict attention to relational structures with a single relation $R$ of arity $r$.

We here reproduce both Herwig's theorem and the HerwigLascar theorem in an argument based on groupoidal constructions, which may offer a starting point for further generalisations. For a specified collection $P$ of partial isomorphisms of $\mathfrak{A}=\left(A, R^{\mathfrak{A}}\right)$, let $S:=\left(\mathbb{Z}_{3}\right)^{P}$ be the set of $P$-indexed tuples over $\{0,1,2\}$. For $p \in P$ we denote as $s \mapsto s+{ }_{p} 1$ the cyclic successor map of $\mathbb{Z}_{3}$ in application to the $p$-th component, which fixes all other components. Consider the vertex set $V:=A \times S=A \times\left(\mathbb{Z}_{3}\right)^{P}$, partitioned according to the natural projections into subsets $V=\bigcup_{s} V_{s}$, where $V_{s}=A \times\{s\}$. We expand $V$ to an $R$-structure $\mathfrak{H}=\left(V, R^{\mathfrak{H}}\right)$ in the natural manner, so that we may think of $\mathfrak{H}$ as a collection of disjoint copies of $\mathfrak{A}$. With $p \in P$ we associate partial bijections $\rho_{p, s}$ from $V_{s}$ to $V_{s+p} 1$

$$
\begin{aligned}
\rho_{p, s}: \operatorname{dom}(p) \times\{s\} & \longrightarrow \operatorname{image}(p) \times\left\{s+_{p} 1\right\} \\
(a, s) & \longmapsto\left(p(a), s+{ }_{p} 1\right) .
\end{aligned}
$$

Putting $E=\left\{\left(s, s+{ }_{p} 1\right),\left(s+{ }_{p} 1, s\right): s \in S, p \in P\right\}$, this structure also gives rise to an $I$-graph $H=\left(V,\left(V_{s}\right),\left(R_{e}\right)\right)$ for $I=(S, E)$, where $R_{\left(s, s+{ }_{p} 1\right)}$ is the graph of $\rho_{p, s}, R_{\left(s+{ }_{p} 1, s\right)}$ its converse. Then the following are symmetries of $H$ and its expansion to the $R$-structure $\mathfrak{H}$ :

$$
\begin{aligned}
\eta_{p}: V & \longrightarrow V \\
(a, s) & \longmapsto\left(a, s-{ }_{p} 1\right),
\end{aligned}
$$

whose induced symmetry of $I$ maps $s \in S=\left(\mathbb{Z}_{3}\right)^{k}$ to $s+{ }_{p} 1$.

Let $\hat{\mathfrak{A}}$ be a realisation of the overlap pattern specified by $H$ that has all the symmetries as described in Corollary IV.4: in particular it lifts the automorphisms $\eta_{p}$ of $H$ to automorphisms of $\hat{\mathfrak{A}}$ and has vertical automorphisms that relate any two hyperedges that project to the same $V_{s}$.

Since any two partition subsets $V_{s}$ of $H$ are related by an automorphism of $H$, any two hyperedges of $\hat{\mathfrak{A}}$ are related by an automorphism of $\hat{\mathfrak{A}}$.

We expand $\hat{\mathfrak{A}}$ to an $R$-structure $\mathfrak{B}:=\left(\hat{A}, R^{\mathfrak{B}}\right)$ by lifting $R^{\mathfrak{A}}$ from every $V_{s}$ to every hyperedge $\hat{s}$ of $\hat{\mathfrak{A}}$ that projects to $V_{s}$ through $\pi_{\hat{s}}: \hat{s} \rightarrow V_{s}$. Then

(i) $\mathfrak{B} \mid \hat{s} \simeq \mathfrak{A}$ for every hyperedge $\hat{s}$ of $\hat{\mathfrak{A}}$;

(ii) the lift of the $\eta_{p}$ to automorphisms of $\hat{\mathfrak{A}}$ are automorphisms also of the $R$-structure $\mathfrak{B}$; so are the 'vertical' automorphisms of $\hat{\mathfrak{A}}$ that relate hyperedges that project to the same $V_{s}$;

(iii) up to suitable 'vertical' automorphisms $\zeta_{p, \hat{s}}, \eta_{p}$ expands the realisation of $p$ in $\pi_{\hat{s}}: \mathfrak{B}\lceil\hat{s} \simeq \mathfrak{A}$ :

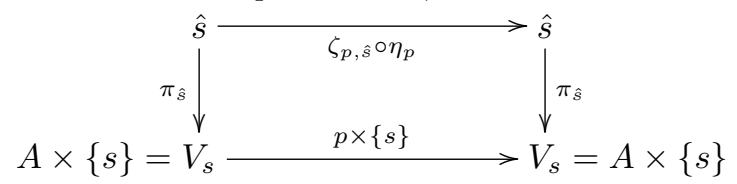

This reproves Herwig's theorem in a form that highlights the role of the hypergraph generated by automorphic images of $\mathfrak{A}$ within the extension $\mathfrak{B} \supseteq \mathfrak{A}$.

Corollary IV.5 (Herwig's Theorem). For every finite relational structure $\mathfrak{A}=\left(A, R^{\mathfrak{A}}\right)$ there is a finite relational structure $\mathfrak{B}=\left(B, R^{\mathfrak{B}}\right)$ and a hypergraph $(B, S)$ such that

(i) $\mathfrak{A} \simeq \mathfrak{B}\lceil s$ for all $s \in S$;

(ii) every partial isomorphism of $\mathfrak{B}$ whose domain and image sets are contained in hyperedges of $(B, S)$ is induced by an automorphism of $\mathfrak{B}$.

This statement can also be obtained as a corollary of the basic form of Herwig's theorem. Its new proof, however, allows for further variations w.r.t. the nature of the hypergraph $(B, S)$, which may for instance be required to be $N$-acyclic. Among other potential generalisations this reproduces the extension of Herwig's theorem to the class of conformal structures and, e.g., of $k$-clique free graphs, obtained on the basis of Herwig's theorem in [10].

As a further consequence of the uniform construction in the new solution to the extension task, we obtain a simpler proof of the Herwig-Lascar theorem. Consider $\mathfrak{A}$ and $P$ as above, together with the $I$-graph $H$ on vertex set $V \times S$ for $S=\left(\mathbb{Z}_{3}\right)^{P}$ and $I=(S, E)$ as above, based on the $\rho_{p, s}$ for $p \in P, s \in S$. Let $\mathfrak{B}$ be obtained, as above, by the natural pull-back of $R^{\mathfrak{A}}$ to a hypergraph $\hat{\mathfrak{A}}=(\hat{A}, \hat{S})$ that realises the overlap pattern specified by the $I$-graph $H$ and is sufficiently symmetric. In particular, for any $\hat{s} \in \hat{S}, \mathfrak{B}\lceil\hat{s} \simeq \mathfrak{A}$, and for every $p \in P$ there is an $f_{\hat{s}, p} \in \operatorname{Aut}(\mathfrak{B})$ that extends $p_{\hat{s}}$, the copy of $p$ in $\mathfrak{B} \mid \hat{s} \simeq \mathfrak{A}$. The following is proved in [14].

Lemma IV.6. Let $\mathfrak{B}^{\prime}$ be any (finite or infinite) solution to the extension task for $\mathfrak{A}$ and $P, \mathfrak{A} \subseteq \mathfrak{B}^{\prime}$. Let $\hat{s}, \hat{s}^{\prime} \in \hat{S}$ and 
$\pi: \mathfrak{B}\left\lceil\hat{s} \simeq \mathfrak{A}\right.$. Then the embedding of $\mathfrak{B}\left\lceil\hat{s}\right.$ into $\mathfrak{A} \subseteq \mathfrak{B}^{\prime}$ via $\pi$ extends to a homomorphism $h: \mathfrak{B}\left\lceil\left(\hat{s} \cup \hat{s}^{\prime}\right) \rightarrow \mathfrak{B}^{\prime}\right.$ :

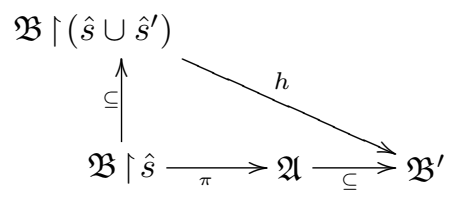

If moreover the hypergraph template $\hat{\mathfrak{A}}=(\hat{A}, \hat{S})$ in the construction of $\mathfrak{B}$ is chosen to be $N$-acyclic, then this local extension process can be iterated to cover any substructure of $\mathfrak{B}$ of size up to $N$, which gives the following.

Corollary IV.7. For any collection $P$ of partial isomorphisms of a finite $R$-structure $\mathfrak{A}$ and for any $N \in \mathbb{N}$, there is a finite extension $\mathfrak{B} \supseteq \mathfrak{A}$ that satisfies the extension task for $\mathfrak{A}$ and $P$ and has the property that any substructure $\mathfrak{B}_{0} \subseteq \mathfrak{B}$ of size up to $N$ can be homomorphically mapped into any other (finite or infinite) solution $\mathfrak{B}^{\prime}$ to the extension task for $\mathfrak{A}$ and $P$.

From our new construction we therefore also obtain the strengthening of Herwig's theorem due to [9], which can be phrased as a finite-model property for the extension task.

Definition IV.8. A class $\mathcal{C}$ of $R$-structures has the finite model property for the extension of partial isomorphisms (EPPA) if, for every finite $\mathfrak{A} \in \mathcal{C}$ and collection $P$ of partial isomorphisms of $\mathfrak{A}$ such that $\mathfrak{A}$ has some (possibly infinite) extension $\mathfrak{A} \subseteq$ $\mathfrak{B} \in \mathcal{C}$ in which each $p \in P$ extends to an automorphism, there is also a finite extension $\mathfrak{A} \subseteq \mathfrak{B}_{0} \in \mathcal{C}$ in which each $p \in P$ extends to an automorphism.

We say that $\mathcal{C}$ is defined in terms of finitely many forbidden homomorphisms if, for some finite list of finite $R$-structures $\mathfrak{C}_{i}$, it consists of all $R$-structures $\mathfrak{A}$ that admit no homomorphisms of the form $h: \mathfrak{C}_{i} \stackrel{\text { hom }}{\longrightarrow} \mathfrak{A}$. The following is now immediate from Corollary IV.7.

Corollary IV.9 (Herwig-Lascar Theorem). Every class $\mathcal{C}$ that is defined in terms of finitely many forbidden homomorphisms has the finite model property for the extension of partial isomorphisms (EPPA).

The guarded fragment $\mathrm{GF} \subseteq \mathrm{FO}$ restricts first-order quantification to variable assignments within guarded subsets; it reflects many of the phenomena known form modal logic at a much higher level of expressiveness and in the richer setting of arbitrary relational structures [2], [7]. Among the most salient features in this direction are its invariance under guarded bisimulation $\sim_{\mathrm{g}}$, which semantically characterises GF as a fragment of FO, see [2], [13]; its finite model property and decidability; and its generalised tree model property [7]. The elegant proof of the finite model property due to [7] uses Herwig's theorem to extend a sufficiently rich finite substructure of an infinite model to a finite model. It turns out that the Herwig-Lascar theorem similarly implies a strengthening of the finite model property for GF first obtained by very different means in [3], in restriction to any class $\mathcal{C}$ defined in terms of finitely many forbidden homomorphisms. We sketch this apparently new and independent argument.

We say that structure $\mathfrak{B}$ is $\sim_{\mathrm{g}}^{\ell}$-homogeneous if any guarded tuples $\mathbf{b}, \mathbf{b}^{\prime}$ in $\mathfrak{B}$ such that $\mathfrak{B}, \mathbf{b} \sim_{\mathrm{g}}^{\ell} \mathfrak{B}, \mathbf{b}^{\prime}$ are related by an automorphism of $\mathfrak{B}$.

Lemma IV.10. Let $\mathcal{C}$ be a class of relational structures defined in terms of finitely many forbidden homomorphisms. Let $\mathfrak{B} \in$ $\mathcal{C}$ be $\sim_{\mathrm{g}}^{\ell}$-homogeneous. Let $\mathfrak{B}^{\prime}$ be the expansion of $\mathfrak{B}$ by a new relation for each one of the finitely many $\sim_{\mathrm{g}}^{\ell}$-types realised in $\mathfrak{B}$. Let $\mathfrak{A}^{\prime}=\mathfrak{B}^{\prime} \uparrow A$ be large enough to contain, for every guarded tuple $\mathbf{b}$ of $\mathfrak{B}$, at least one realisation of that $\sim_{\mathrm{g}}^{\ell}$ type. Then $\mathfrak{A}^{\prime}$ has a Herwig extension $\overline{\mathfrak{A}}^{\prime} \supseteq \mathfrak{A}^{\prime}$ in $\mathcal{C}$ that is $\sim_{\mathrm{g}}$-equivalent to $\mathfrak{B}^{\prime}$ in the sense that $\overline{\mathfrak{A}}^{\prime} \sim_{\mathrm{g}} \mathfrak{B}^{\prime}$ and $\overline{\mathfrak{A}}^{\prime}, \mathbf{a} \sim_{\mathrm{g}}$ $\mathfrak{B}^{\prime}, \mathbf{a}$ for every guarded tuple $\mathbf{a} \in \mathfrak{A}$.

Let $\mathcal{C}$ be defined by the condition that there are no homomorphic images of the finite structures $\mathfrak{C}_{1}, \ldots, \mathfrak{C}_{m}$. The class $\mathcal{C}_{0} \supseteq \mathcal{C}$ of structures that admit no acyclically embedded homomorphic images of the $\mathfrak{C}_{i}$ is definable in $\mathrm{GF}$ by some $\gamma \in \mathrm{GF}$. To find finite models of $\varphi \in \mathrm{GF}$ in $\mathcal{C}$, choose $\ell$ greater or equal to the nesting depth of $\varphi \wedge \gamma$. If $\varphi$ has an infinite model in $\mathcal{C}$, then $\mathrm{a} \sim_{\mathrm{g}}^{\ell}$-homogeneous infinite model $\mathfrak{B}$ of $\varphi$ in $\mathcal{C}$ can be obtained as a suitable regular tree-like model of $\varphi \wedge \gamma$ (which in turn could be obtained from any finite model of $\varphi \wedge \gamma$ ). The lemma guarantees a finite model in $\mathcal{C}$. So we obtain the following result from [3].

Corollary IV.11. GF has the finite model property over any class defined in terms of finitely many forbidden homomorphisms.

\section{REFERENCES}

[1] N. Alon. Tools from higher algebra. In R. Graham, M. Grötschel, and Lovász, editors, Handbook of Combinatorics, volume II, pages 17491783. North-Holland, 1995.

[2] H. Andréka, J. van Benthem, I. Németi. Modal languages and bounded fragments of predicate logic. Journal of Philosophical Logic, 27, 1998: 217-274.

[3] V. Bárány, G. Gottlob, M. Otto. Querying the guarded fragment Accepted for Logical Methods in Computer Science, 2012.

[4] C. Beeri, R. Fagin, D. Maier, and M. Yannakakis. On the desirability of acyclic database schemes. Journal of the ACM, 30:497-513, 1983.

[5] C. Berge. Graphs and Hypergraphs. North-Holland, 1973.

[6] N. Biggs. Cubic graphs with large girth. In G. Blum et al., eds, Annals of the New York Academy of Sciences, volume 555, pages 56-62. 1989.

[7] E. Grädel. On the restraining power of guards. Journal of Symbolic Logic, 64, 1999: 1719-1742.

[8] B. Herwig. Extending partial isomorphisms on finite structures. Combinatorica, 15:365-371, 1995.

[9] B. Herwig and D. Lascar. Extending partial isomorphisms and the profinite topology on free groups. Transactions of the AMS, 352:19852021, 2000.

[10] I. Hodkinson and M. Otto. Finite conformal hypergraph covers and Gaifman cliques in finite structures. Bulletin of Symbolic Logic, 9:387405, 2003.

[11] E. Hrushovski. Extending partial isomorphisms of graphs. Combinatorica, 12:411-416, 1992.

[12] M. Otto. Modal and guarded characterisation theorems over finite transition systems. Annals of Pure and Applied Logic, 130:173-205, 2004.

[13] M. Otto. Highly acyclic groups, hypergraph covers and the guarded fragment. Journal of the ACM, 59 (1), 2012.

[14] M. Otto. On groupoids and hypergraphs. Technical report on arXiv:1211.5656, 2012. 